\title{
UTILIZAÇÃO DE METILFENIDATO, LISDEXANFETAMINA E MODAFILINA COMO DROGAS AMPLIADORAS DO CONHECIMENTO: ESTUDO DO PERFIL DE DISPENSAÇÃO EM UMA FARMÁCIA COMUNITÁRIA DO INTERIOR DO RIO DE JANEIRO
}

\author{
Cristiano Guilherme Alves de OLIVEIRA*, Natalha Felisbino RIBEIRO, Juliano Gomes BARRETO, Denise \\ Aparecida da SILVA \& Rondinelli de Carvalho LADEIRA
}

Universidade Iguaçu - Campus V - Faculdade de Farmácia. Itaperuna, Rio de Janeiro, Brasil.

*Autor para correspondência: cristiano.farma@ hotmail.com

DOI: http://dx.doi.org/10.18571/acbm.130

\section{RESUMO}

Os ampliadores cognitivos trazem uma possível melhora na capacidade da atenção, memória e no estado de alerta. No entanto, a utilização de fármacos para tal finalidade pode apresentar riscos para a saúde apresentando uma forma irracional da utilização destes medicamentos. Desta forma, foi realizado uma pesquisa na dispensação de medicamentos metilfenidato, lisdexanfetamina e modafilina em uma farmácia comunitária, através do balanço de medicamentos registrados no SNGPC nos anos de 2016 e 2017. Os dados observados descrevem uma sazonalidade da venda destes medicamentos com predominância de metilfenidato de $10 \mathrm{mg}$ para apresentação de 30 e 60 comprimidos. A prescrição dos fármacos acontece por grande maioria por especialistas (psiquiatras), ocorrendo uma parte de não especialistas. Ocorre um aumento significativo do consumo destes medicamentos entre os anos de 2016 e 2017. Alertas da ANVISA assim como dados obtidos no trabalho sugerem a necessidade de alerta para o consumo destes medicamentos de forma irracional.

Palavras chave: Ampliadores cognitivos; Metilfenidato; Uso irracional.

\begin{abstract}
Cognitive enhancers bring about a possible improvement in attention, memory, and alertness. However, the use of drugs for this purpose may present health risks by presenting an irrational use of using these drugs. Thus, a study was carried out in the dispensation of methylphenidate, lisdexamphetamine and modafilin in a community pharmacy, through the balance of medicines registered in the SNGPC in the years 2016 and 2017. The observed data describe a seasonality of the sale of these drugs with predominance of methylphenidate $\mathrm{Of} 10 \mathrm{mg}$ for presentation of 30 and 60 tablets. The prescription of the drugs happens by great majority by specialists (psychiatrists), occurring a part of nonspecialists. There is a significant increase in the consumption of these drugs between the years 2016 and 2017. ANVISA alerts as well as data obtained in the study suggest the need to alert consumers to the consumption of these drugs in an irrational use.
\end{abstract}

Keywords: Cognitive enhancers; Methylphenidate; Irrational use.

\section{Introdução}

As drogas amplificadoras da capacidade cognitiva têm sido usadas em idosos, pessoas com capacidade neurodegenerativa e demência para uma melhora na qualidade de vida. Ao passo, a utilização dessas drogas por estudantes sadios vem aumentando de forma considerável. Tais práticas visam à busca da melhora cognitiva, emocional e como prolongadores do estado de vigília (BATISTELA et al., 2014). 
No entanto, A utilização dessas drogas de forma irracional pode provocar efeitos tóxicos a curto e longo prazo. Levando a necessidade de se aumentar o debate sobre o crescimento do número de prescrição e dispensação de Metilfenidato a ANVISA emite nota em 2012 para o alerta de se aumentar a necessidade de monitoramento de drogas que podem provocar dependência física e psíquica. Afirma a Agência Nacional que os dados de prescrição e do consumo de medicamentos podem ser utilizados no monitoramento de riscos sanitários e no planejamento e na avaliação do progresso em saúde de uma comunidade (ANVISA, 2012).

De acordo com ANVISA (2013) dados recentes divulgados pela Agência mostram o uso crescente do medicamento em todas as regiões do país. Considerando-se o indicador DDD (dose diária definida)/1000 crianças entre 6 e 16 anos/dia, o aumento no consumo do fármaco foi de $164 \%$ entre 2009 e 2011. No boletim farmacoepidemiológico do SNGPC é destacado o caráter socioantropológico, comportamental e comercial na utilização de fármacos. Destaca-se ainda que Os estudos de utilização de medicamentos (EUM) objetivam, segundo a Organização Mundial da Saúde (OMS), estudar a comercialização, distribuição, prescrição e uso de medicamentos na sociedade com ênfase especial nas consequências médicas, sociais e econômicas (ANVISA, 2012).

Além dos boletins citados pelo órgão regulador tem-se uma série de autores que descrevem os potenciais abusos na utilização irracional dos fármacos descritos. Como Brant e Carvalho (2012) que descrevem a utilização de Metilfenidato como uma invenção da contemporaneidade como um gadget com objeto de consumo curto e rápido, parceiro conectável e desconectável ao alcance das mãos, tornando-se dispositivo de prazeres efêmeros, fabricado e comercializado como medicamento.

Masías-Arias e Fiestas-Teque (2014) apresentam um transtorno psicótico de um adolescente de 14 anos que alucinações auditivas, após o intervalo de quatro dias de medicação com metilfenidato. Corrobora também Rapello et al. (2015) ao anunciarem que efeitos adversos podem ser apresentados como insônia, dores abdominais e anorexia. Os autores descreveram que em altas doses o metilfenidato pode provocar enfisema pulmonar em ratos.

Tendo como contraponto a utilização destes fármacos como "muletas" cognitivas autores Caliman e Rodrigues (2014) apontam para o aumento do diagnóstico do Transtorno de Déficit de Atenção e Hiperatividade (TDAH) descrito como um dos diagnósticos que mais sustentam o processo atual de medicalização da vida, devido ao crescente consumo de metilfenidato. Com experiência de tratamentos em adultos não tratados na infância ou adolescência.

Como ponto de partida os fatos citados, o artigo tem como foco trazer a luz do tema um estudo de caráter exploratório de cunho epidemiológico sobre a utilização destes fármacos. Assim, objetivou-se identificar o perfil da dispensação de fármacos "promotores cognitivos" como: Metilfenidato, Modafilina e Lisdexanfetamina em uma farmácia comunitária em Itaperuna-RJ.

\section{Metodologia}

Os dados foram colhidos no intervalo de janeiro de 2016 a abril de 2017 períodos de abertura de uma grande loja da rede Pague Menos na cidade de Itaperuna-RJ. Os dados obtidos representam as receitas aviadas e dispensadas junto ao programa informatizado da rede que integra o Sistema Nacional de Gerenciamento de Produtos Controlados (SNGPC) como forma de boletim mês a mês onde variáveis como: fármaco, formulação, quantidades de caixas prescritas, quantidades de caixas dispensadas e especialidades médica foram obtidas e plotadas em tabelas e gráficos. Os dados foram tabulados em planilha Excel $^{\circledR}$ e tratados estatisticamente para a análise de variância pelo método de ANOVA com intervalo de confiança de 95\%. Foram usados para avaliação da progressão das vendas estatística descritiva e métodos de regressão linear. 


\section{Biomedica Brasiliensia}

\section{Resultados}

A utilização de fármacos que causam dependência ou alteradores da consciência estão sujeitos ao controle da protaria 344/98. Desta forma, os EUM podem ser realizados através do inventário emitido por programas de controle do SNGPC que permitem a observação da quantidade de farmacos prescritos e suas formulações de acordo com a especialidade medica durante um lapso temporal pré determinado pelos pesquisadores.

Para a observação da dispensação de medicamentos em sua totalidade no intervalo de tempo de 2016 (ao fim de um ano) obteve-se a figura 1 representando a evolução da dispensação de Metilfenidato, Lisdexanfetamina e Modifilina mês a mês.

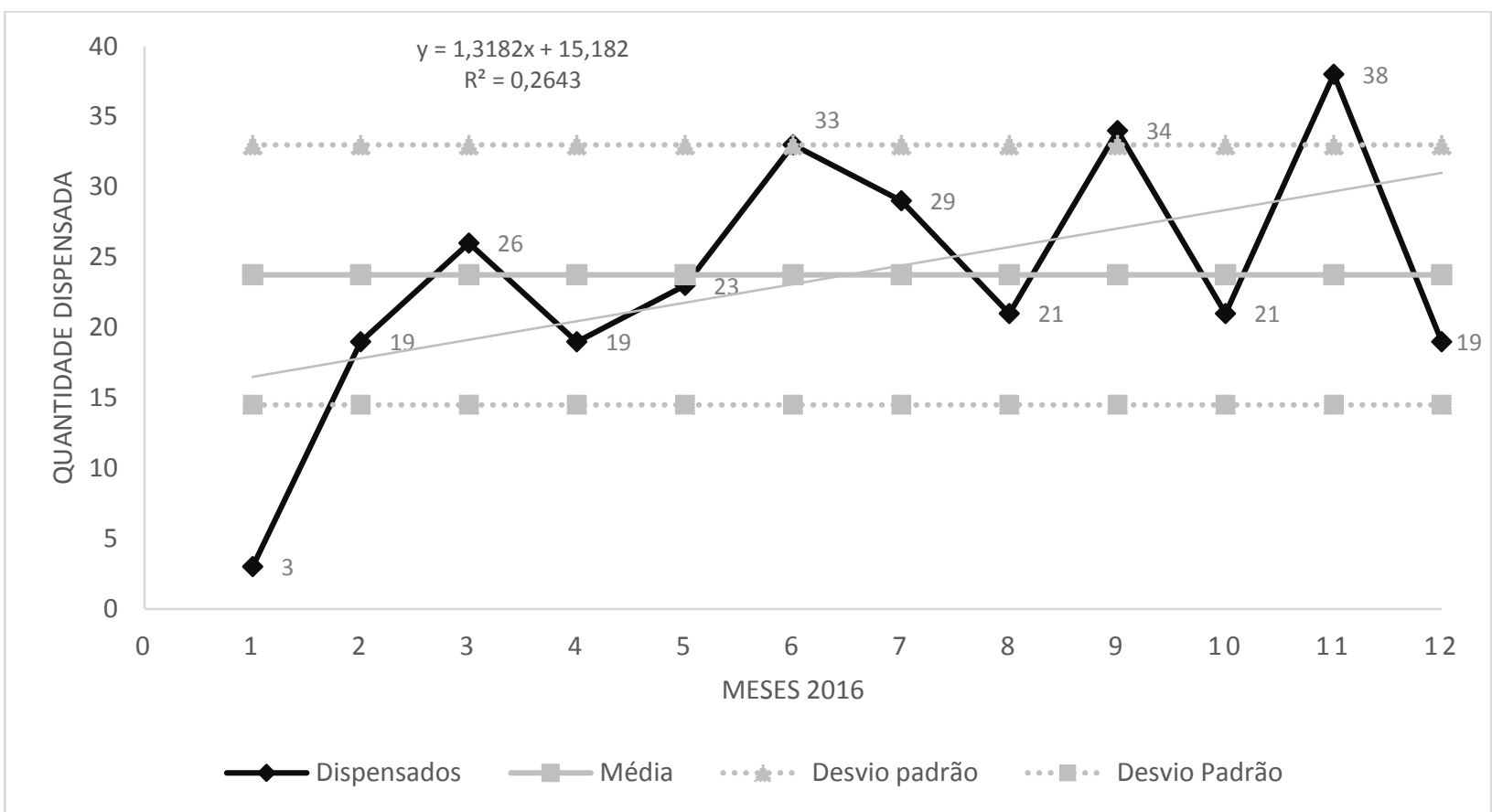

Figura 1: Dispensação total por unidade de caixa de Metilfenidato, Lisdexanfetamina e Modafilina em 2016 (média= 23,75 e desvio padrão=9,24).

Em relação aos medicamentos dispensados por principio ativo e formulação tem-se a tabela (1), onde as variáveis são tratadas sobre a comparação das variâncias pelo método de ANOVA onde Fcrítico = 2,7173 e Fcalculado = 5,2839 com valor de $\mathrm{P}=0,003972$. Ao rejeitar a hipótese de nulidade entre as variâncias submete-se a amostra ao teste TUKEY com IC(95\%) e obteve-se a Diferença Mínima Significativa $(\mathrm{DMS})=41,447$ onde temos o elenco determinado pelas letras a e b onde as variáveis que apresentam a mesma denominação são iguais e representadas por letras diferentes na DMS são diferentes no escalonamento. 
Tabela 1: Dispensação de medicamentos por princípio ativo e formulação.

\begin{tabular}{ccccccc}
\hline Medicamento & $\mathbf{2 0 1 6}$ & $\mathbf{2 0 1 7}$ & Soma & Média & Tukey & DMS \\
\hline Metilfenidato 10mg/30 & 133 & 62 & 195 & 97,5 & 33,5 & $\mathrm{~A}$ \\
Metilfenidato 10mg/60 & 94 & 34 & 128 & 64 & 62,5 & $\mathrm{~A}$ \\
Metilfenidato 10mg/30LA & 3 & 0 & 3 & 1,5 & 1 & $\mathrm{~B}$ \\
Metilfenidato 20mg/30LA & 1 & 0 & 1 & 0,5 & 0 & $\mathrm{~B}$ \\
Metilfenidato 40mg/30LA & 1 & 0 & 1 & 0,5 & -4 & $\mathrm{~B}$ \\
Metilfenidato 18mg/30 & 6 & 3 & 9 & 4,5 & 2,5 & $\mathrm{~B}$ \\
Metilfenidato 36mg/30 & 4 & 0 & 4 & 2 & 0,5 & $\mathrm{~B}$ \\
Modafinila 100mg/30 & 3 & 0 & 3 & 1,5 & $-2,5$ & $\mathrm{~B}$ \\
Modafinila 200mg/30 & 5 & 3 & 8 & 4 & $-14,5$ & $\mathrm{~B}$ \\
Lisdexanfetamina 30mg/28 & 18 & 19 & 37 & 18,5 & 8,5 & $\mathrm{~B}$ \\
Lisdexanfetamina 50mg/28 & 10 & 10 & 20 & 10 & 7,5 & $\mathrm{~B}$ \\
Lisdexanfetamina 70mg/28 & 3 & 2 & 5 & 2,5 & 2,5 & $\mathrm{~B}$ \\
\hline
\end{tabular}

Para a avaliação da variação do aumento da dispensação dos medicamentos obteve-se a comparação entre os quatro primeiros meses dos anos de 2016 e 2017 por número de caixas do total dos medicamentos. A análise permite a tabulação de dois gráficos, demonstrados nas figuras 2 e 3, dados de forma total e separados mês a mês. Na totalidade pode-se observa um aumento da dispensação dos quatro primeiros meses de 98,50\% na dispensação das drogas estudadas

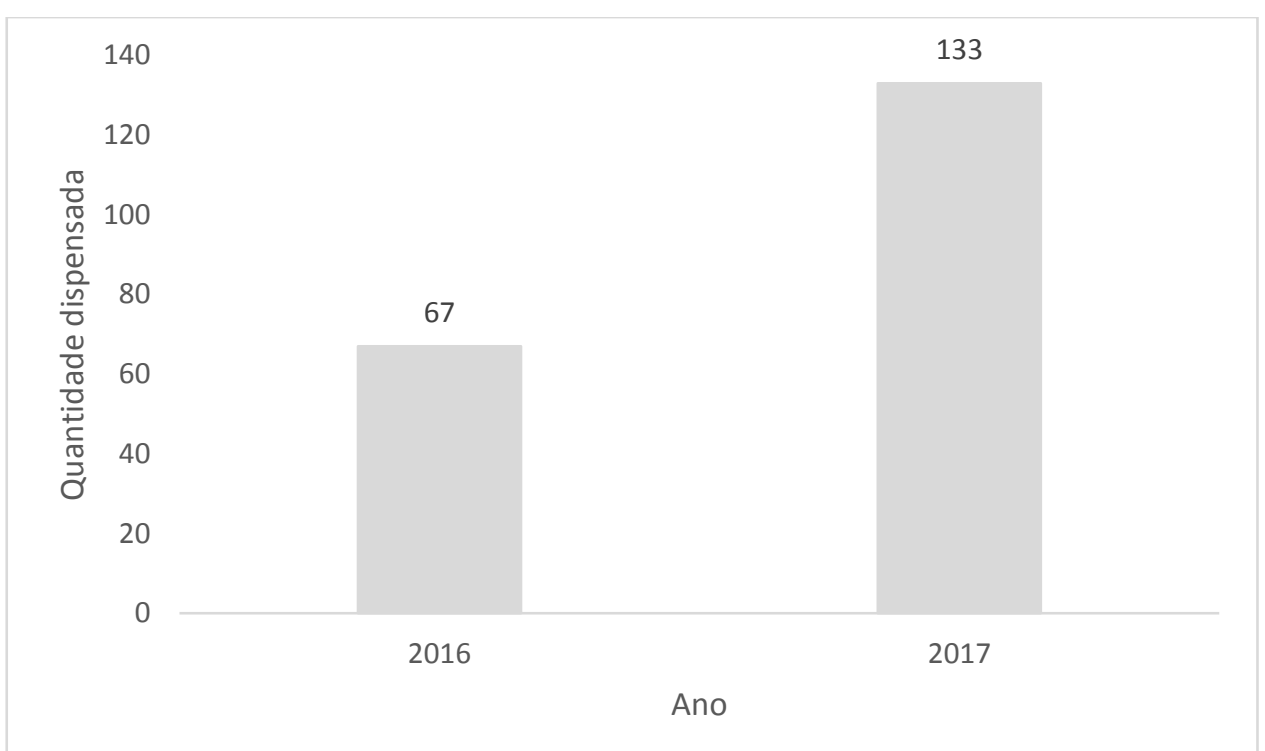

Figura 2: Quantidade total de medicamentos dispensados nos quatro primeiros meses de 2016 e 2017. 


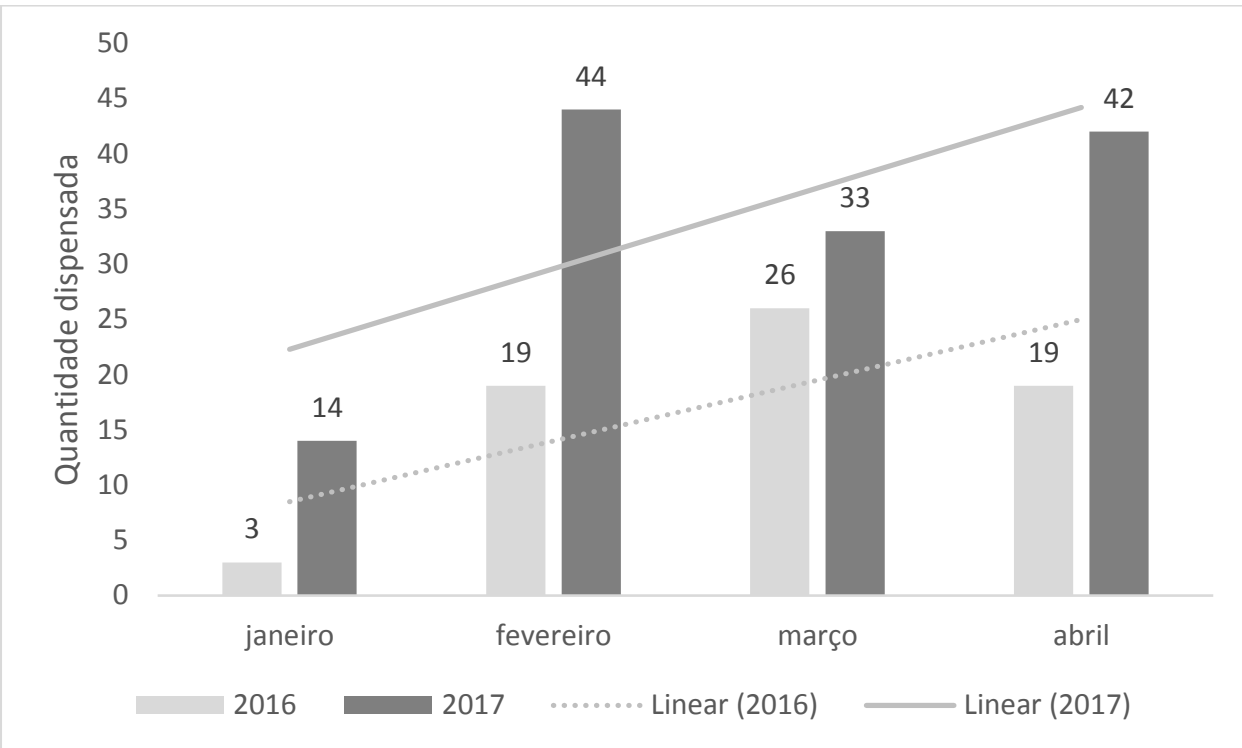

Figura 3: Dispensação mde medicamentos mês a mês dos quatro primeiro mese de 2016 e 2017.

Para a compreensão do perfil da dispensação por especialidades tem-se na figura 4 onde a totalidade de caixas dispensados de todos os medicamentos estudados foram divididos em anos de 2016 e 2017 e relacionados a cada especialidade médica de acordo com a descrição na recita do CRM de registro do prescritor.

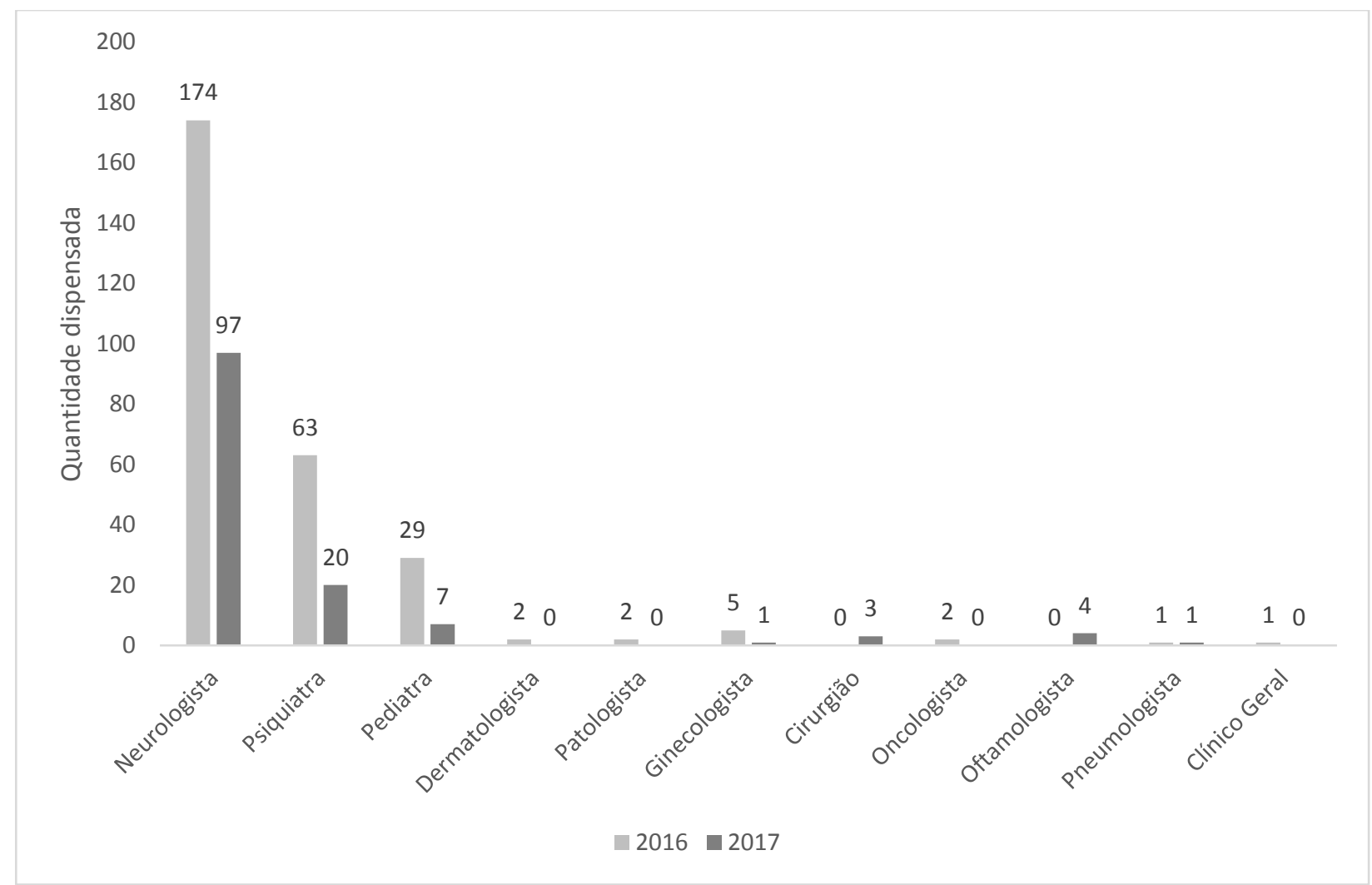

Figura 4: Dispensação de medicamentos por especialidades médicas.

O tratamento estatístico para a variância em relação às especialidades médicas pelo método de ANOVA com IC (95\%) obteve-se F crítico $=2,853625$ e F calculado $=8,766233766$ demonstrando uma rejeição da hipótese de nulidade com variação da dispensação de especialistas 
para a dispensação que submetidos a método de TUKEY e plotados na tabela (2) com $\mathrm{DMS}=42,75543$.

Tabela 2: Diferença estatística da dispensação por especialidade médica pelo método de TUKEY.

\begin{tabular}{ccccc}
\hline Grupo & Contagem & Soma & Média & DMS \\
\hline Neurologista & 2 & 271 & 135,5 & $\mathrm{~A}$ \\
Psiquiatra & 2 & 83 & 41,5 & B \\
Pediatra & 2 & 36 & 18 & B \\
Dermatologista & 2 & 2 & 1 & B \\
Patologista & 2 & 2 & 1 & B \\
Ginecologista/obstetra & 2 & 6 & 3 & B \\
Cirurgião plástico & 2 & 3 & 1,5 & B \\
Oncologista & 2 & 2 & 1 & B \\
Oftalmologista & 2 & 4 & 2 & B \\
Pneumologista & 2 & 2 & 1 & B \\
Clínico geral & 2 & 1 & 0,5 & B \\
\hline
\end{tabular}

\section{Discussão}

A modernidade trás a utilização de substâncias que elevam a capacidade produtiva. Este tipo de consumo tem sido de forma crescente observado no Brasil e no mundo. Com anseio no reconhecimento social do individuo, para fins instrumentais na produção ou de forma recreativa vem se impulsionando o uso e a prescrição farmacológica legal destas drogas. A medicalização torna ramos da psiquiatria uma ciência capaz de tratar distúrbios além da loucura com a valorização do individuo e o bem-estar sempre de bom humor para os indivíduos (BRANT e CARVALHO, 2012).

No mesmo sentido, tais estudos podem apontar para o aumento do acesso de medicamentos à população. A melhoria nas técnicas de diagnóstico do Transtorno de Déficit de Atenção e Hiperatividade (TDAH) pode produzir aumento crescente do consumo de fármacos como metilfenidato. Embora autores como Calimam e Rodrigues (2014) abordem a medicalização como questões problemáticas como: o número exagerado de diagnósticos de TDAH em crianças e adultos; a patologização de questões de cunho educacional e a expansão da utilização não médica destes fármacos.

Para tal os EUM trazem um papel na observação da tendência dessa medicalização e traça perspectivas para o uso racional do medicamento. Demostrando através de dados epidemiológicos a análise comportamental da utilização de fármacos pelo método de inventário de vendas com prescrições do SNGPC.

De acordo com a Associação Brasileira do Déficit de Atenção, os medicamentos recomendados em consensos de especialistas são os estimulantes (lisdexanfetaminae e metilfenidato), a modafinila apresenta se como fármaco no tratamento da narcolepsia. Tais escolhidos pelos para a pesquisa de ampliadores cognitivos.

Assim no presente estudo, a observação da prescrição de fármacos com metilfenidato, modafinila e Lisdexanfetaminas em sua totalidade foi distribuída mês a mês no ano de 2016 como observados no gráfico (1) com duas linhas de tendência sendo a média (primeira linha) e média somada com o desvio padrão (segunda linha). Observa-se que nos meses de Abril, Setembro e Novembro o consumo das drogas sobem acima do valor da segunda linha de tendência média mais desvio padrão mostrando uma sazonalidade no perfil das vendas. Assim sendo, o aumento ocorre com uma aproximação do calendário letivo de provas da cidade de Itaperuna no interior do Rio de 
Janeiro cidade reconhecida por albergar faculdades com cursos na área de Medicina, Medicina Veterinária, Odontologia, Farmácia, e demais cursos das áreas de humanas, saúde e exatas. O aumento da demanda bimestral dessas drogas, aqui demostrada no trabalho como utilização de ampliadores cognitivos demostra uma forma de uso irracional dos medicamentos já que estudos não apontam para a melhora cognitiva em jovens saudáveis.

Autores como Freese et al. (2012) reportam a necessidade de se identificar o aumento do uso não médico de metilfenidato. No Brasil, as resoluções federais no. 344/1998 e 22/2001 incluem estas substâncias na categoria A3 (substâncias psicotrópicas) e estão sujeitas a prescrição controlada como resultado do alto risco de dependência química. Ainda os mesmos autores descrevem ampla revisão de literatura onde afirmam que, no entanto, as evidências não apoiam a conclusão de que eles possam promover um melhor desempenho cognitivo.

Freese et al. (2012) descrevem em seu estudo o potencial de abuso do metilfenidato que em altas doses podem produzir efeitos recompensadores ou reforçadores subjetivos, semelhantes aos da cocaína, fazendo com que o indivíduo se sinta euforizado (ou alto). Ao nível comportamental do abuso de substâncias, o reforço é referido como um dos aspectos mais determinantes da toxicodependência, sugerindo que as drogas que induzem este mecanismo têm um maior potencial de abuso.

Autores como Batistela et al. (2014) descrevem estudo realizado em 36 jovens universitários sobre o uso de metilfenidato com 10, 20 e $40 \mathrm{mg}$ que se submeteram a testes cognitivos, memória operacional, episódica e testes de atenção. Não foram observadas diferenças estatísticas entre o grupo de controle sobre o uso de placebo e os estudantes sobre o uso de metilfenidato. Sendo relatada a melhora do bem-estar em doses acima de 40mg. A melhora do bem-estar associada ao ganho na produtividade escolar.

Tais relatos de sazonalidade do presente trabalho, em relação à dispensação de drogas ampliadores cognitivas coincidem com os dados obtidos pelo Boletim Brasileiro de Avaliação em tecnologias da Saúde (BRAT) a partir dos dados registrados no SNGPC que mostram também o aumento no consumo de metilfenidato no País tem comportamento variável, com destaque para redução nos meses de férias e aumento no segundo semestre dos anos estudados (BRATS, 2014). Tais dados de aumento no segundo trimestre na dispensação destes fármacos são observados nos anos de 2009 e 2011 pelo boletim de farmacoepidemiologia do SNGPC em 2012 (ANVISA, 2012).

Em relação à Dose Diária Definida (DDD) para metilfenidato o valor obtido foi de 0,0957 DDD/1000 habitantes por dia, valor considerado para uma farmácia no universo de 80 estabelecimentos na cidade de Itaperuna- RJ no ano de 2016. Para o cálculo de uma população de 99507 habitantes (IBGE, 2017). Com a DDD recomendada para estudo de $30 \mathrm{mg} / \mathrm{dia}$. Trabalho realizado por Perini et al. (2014) em farmácias de Belo Horizonte no ano de 2006 obtiveram o valor de $0.37 \mathrm{DDD} / 1000$ habitantes por dia, os autores trazem valores de outros países como Espanha 1,26 DDD/1000habitantes/dia e Chile 0,56 DDD/1000habitantes. Desta forma, o valor de DDD para cidade de Itaperuna fica subdimensionado por não totalizar a quantidade total de metilfenidato dispensado no ano de 2016. Mas pode demostrar um alerta de uma movimentação elevada de metilfenidato por dia na cidade. Não foram feitos cálculos de DDD para Lisdexanfetaminas e Modafinila.

De acordo com as formulações e especialidades dos ampliadores cognitivos pode-se observar na tabela (1) uma venda maior de metilfenidato $10 \mathrm{mg} / 30$ e $10 \mathrm{mg} / 60$ comprimidos com a diferença estatística pelo método de TUKEY e IC (95\%) sem diferenças entre as demais especialidades e formulações. A formulação com $10 \mathrm{mg}$ com 30 e 60 comprimidos aparece com opções mais dispensadas. Sendo que as duas modalidades citadas representam um total de 93,4\% das amostras dispensadas em 2016 e $72 \%$ das vendas no ano de 2017 . Tal valor depara-se com o aumento de Lisdexanfetamina de 8,67\% em 2016 para 23,30\% considerando somente os quatro primeiros meses de 2017. A Modafilina com baixo percentual de utilização em ambos os anos. 
A Lisdexanfetamina (LDX) representa uma pró-droga, sendo metabolizada no sangue pela enzima L-lisina em d-anfetamina sendo um processo lento que permite o aumento da meia vida do fármaco. Porem, um importante fator para o não desenvolvimento de dependência e abuso destes fármacos após a utilização oral comparada as demais anfetaminas o que permite explicar o crescimento das vendas no ano de 2017. Mattos (2014) ressalta que a LDX possui eficácia comparável ou superior à dos demais psicoestimulantes disponíveis. $\mathrm{O}$ autor documenta eficácia em longo prazo, com perfis de segurança e tolerabilidade comparáveis aos dos demais estimulantes usados no tratamento do TDAH. A maioria dos eventos adversos associados à LDX é considerada leve ou moderada quanto à gravidade, sendo os eventos mais comuns: perda de apetite e insônia.

Pineda (2014) no boletim sobre a utilização da LDX em Castillha afirma o seu potencial em tratar casos de TDAH com resultado semelhante aos metilfenidatos de liberação prolongada. Também ressalta efeitos como insônia, perda do apetite, boca seca, cefaléia e dor abdominal. Alerta para a diferença considerada de preço entre os fármacos. Sendo o valor da Lisdexanfetamina é muito superior do que o metilfenidato.

É de se destacar que a insônia é o efeito mais proeminente na literatura sobre o abuso e utilização clinica desses fármacos, sendo um fator fundamental para a utilização não médica ou irracional dessa medicação. As grandes cargas laborais somadas a jornadas excessivas de aulas e trabalhos com calendários curtos e a movimentação "Fast Food" que impõe a modernidade, permite a essa pratica considerada como "arrebite" para o ajuste da rotina dessas demandas cada vez mais crescentes.

Magalhães (2014) afirma que drogas (no sentido de medicamentos) tais como Modafinil, Metilfenidato e outras, são usadas "off-label" de forma a aumentar a capacidade de concentração, aprendizado e facilitar a retenção de informação em um indivíduo. O autor afirma que Substâncias que agem no sistema dopaminérgico (aumentando à concentração do neurotransmissor a dopamina no cérebro) como o Modafinil e o Metilfenidato conseguem modular alguns aspectos da memória de trabalho de um indivíduo. Trata o autor essas drogas ampliadoras cognitivas pelo termo de "smart drugs".

Interessante notar que das drogas apresentadas neste trabalho a de menor dispensação a modafilina é que para Magalhães (2014) apresenta melhor desempenho como "smart drugs". O autor traz um trabalho em que demonstra em uma larga bateria de testes, o modafinil foi responsável por aumentar a memória de reconhecimento de padrões visuais, planejamento espacial e tempo de reação/latência em diferentes tarefas ligadas à memória de trabalho sendo apelidada popularmente como "pílula da inteligência".

Sendo usado no tratamento de narcolepsia é usado em outros casos de sonolência excessiva e pode ser usado de modo a abrandar a diminuição de desempenho devido ao sono reduzido aparentemente com efeitos colaterais muito baixos e poucos riscos de dependência. É sabido, também, através de outros trabalhos, que estimula a neurogênese na região do hipocampo. O nome comercial, no Brasil, é Stavigile (MAGALHÃES, 2014).

Em estudo realizado por Wesensten (2006) traz a utilização do modafinil como importante na melhora do desempenho para indivíduos com privação do sono. E alerta para níveis de privação de sono. Modafinil também prejudica pós-sono privação recuperação sono, mas de poucos Estudos disponíveis sobre esta questão, não está claro se essas deficiências de sono se traduzem em desempenho pós-sono.

Pode-se observar de acordo com gráfico 2 e 3, um aumento de 98,50\% das dispensações de fármacos estudados em relação aos quatro primeiros meses do ano de 2016 para 2017. Observase que existe uma sazonalidade bimestral no aumento da dispensação nos meses de fevereiro e abril consequente aos períodos letivos de inicio do semestre letivo e aos calendários de provas. Tais achados demonstram um alerta para o aumento do consumo destas substâncias representando o dobro dos medicamentos vendidos no intervalo de 2016. Desta forma, a DDD de metilfenidato para o ano de 2017 no valor de 0,03659 se manifesta superior ao valor de DDD de 2016 de 0,0229 
apresentando um aumento de 59,03\%. Agência Nacional de Vigilância Sanitária (ANVISA) apontam para o uso crescente do medicamento em todas as regiões do país (ANVISA, 2013). Considerando-se o indicador DDD (dose diária definida) /1000 crianças entre 6 e 16 anos/dia, o aumento no consumo do fármaco foi de $164 \%$ entre 2009 e 2011.tais valores do aumento podem ser mascarados pelo aumento da dispensação de LDX e modafilina nos anos de 2017. Não foram calculados neste trabalho valores para DDD de LDX e modafilina.

Em relação às especialidades médicas dos prescritores pode-se observar uma prevalência estatística da prescrição de neurologistas pelo método de TUKEY com IC (95\%) em relação às demais especialidades. Representando 65,77\% das dispensações. As demais seguidas de 20,14\% de neurologistas, $8,73 \%$ de pediatras e 5,33\% de demais especialidades como Cirurgião plástico, oftalmologistas, pneumologistas, clínico geral, patologistas.

De acordo com Walylo et al. (2016) tem-se que as principais normas vigentes para prescrição e dispensação são: as leis Federais 5.991/73 e 9.787/99; Resolução 357/2001, do Conselho Federal de Farmácia; Resoluções 1.552 (de 20/08/99), 1.477 (de 11/7/97) e 1.885 (de 23/10/08) do Conselho Federal de Medicina (CFM). Sendo que a portaria MS/ SVS n ${ }^{\circ} 344$, de 12 de maio de 1998 "as substâncias entorpecentes e psicotrópicas exigem formulários de receita específica (Notificações de Receita A e B)". As drogas apontadas neste estudo necessitam de talonário especial tipo A é de cor amarela e é usada para a prescrição dos medicamentos presentes nas listas A1, A2 e A3, entorpecentes e psicotrópicos. A "Notificação de Receita A" tem validade por 30 (trinta) dias, a partir da data de sua emissão e é válida em todo território nacional. É fornecida de forma numerada e controlada pela Autoridade Sanitária Estadual ou pelo Distrito Federal, gratuitamente, aos profissionais e instituições cadastradas, em talonário de 20 folhas.

Registre-se ainda, que o medicamento deve ser prescrito com a quantidade máxima para 30 dias de tratamento. Conforme posologia registrada na notificação e tendo validade em todo território nacional. Havendo necessidade de uma quantidade superior de medicamentos, a receita necessita de uma justificativa do uso. Em uma prescrição médica deve conter informações fundamentais sobre o medicamento, bem como, o nome do medicamento em letra legível, a dose, posologia, duração do tratamento, via de administração, data da prescrição, nome do paciente, nome do médico prescritor e o registro no Conselho Regional de Medicina (ANVISA, 2016).

Ferreira (2014) ressalta ainda em parecer do Conselho Federal de Medicina (CFM) que se pode observar, não há vedação expressa em nenhum dos pareceres, leis e documentos apontados com relação à prescrição para o próprio prescritor, exceto no caso de autoprescrição de substâncias entorpecentes e psicotrópicos, conforme disposto no art. 21 do Decreto-lei no 20.931/3.

Traz ainda o autor que é de uso obrigatório do carimbo assinalado na Portaria no $344 / 98$ só se dá no $\S 2^{\circ}$ do art. 40 para recebimento do talonário para prescrição de medicamentos e substâncias das listas A1 e A2 (entorpecentes) e A3 (psicotrópicos).

De acordo com CRF-SP (2017) a receita deverá ser remetida até o dia 15 do mês subsequente às Autoridades Sanitárias Estaduais ou Municipais e do Distrito Federal, por relação em duplicata, que será recebida pela Autoridade Sanitária competente mediante recibo, a qual, após conferência, será devolvida no prazo de 30 dias. Não esta explicitada nas legislações acima citadas à necessidade de especialidade em psiquiatria ou neurologia para a obtenção de do talonário especial para receita amarela.

Em alerta terapêutico da ANVISA (2014) segue as orientações para os prescritores antes de prescrever metilfenidato, verifique se o paciente possui histórico pessoal ou familiar de doenças cardiovasculares (taquicardia, hipertensão, infarto, derrame e morte súbita), problemas psiquiátricos (convulsões e doenças psicóticas, pois o paciente pode apresentar piora ou alteração no comportamento, depressão, ideação suicida, bipolaridade, agressividade e alucinações) ou abuso/dependência de álcool, drogas, benzodiazepínicos e outros medicamentos com ação no sistema nervoso central; 
Apesar de Carlini et al. (2003) descreverem em seu trabalho que tais fármacos seriam prescritos somente por especialistas e não possuírem potencial de abuso. Fato não observado nesse trabalho, onde parte significativa dos prescritores maior que $14 \%$, considerando pediatras como não especialidades para prescrição de TDAH, são responsáveis diretos para a utilização desses fármacos. Em alerta terapêutico a ANVISA (2014) relata que a maioria dos prescritores são psiquiatras e neurologistas, mas havendo um número considerável de prescritores não especialistas.

Em relação à dependência e potencial de abuso em adultos que foram submetidos na infância ao metilfenidato, Jaboinski et al. (2015) descreve a necessidade de se observar estudos mais profundos que possam afastar essa hipótese e aponta para o risco de adultos com propensão ao abuso de psicoestimulantes. Considerando seu alto potencial de abuso e dependência, torna-se premente a adoção de debates que abordem a atual problemática do consumo indevido do metilfenidato, alertando a população para o mau uso, os efeitos adversos e as consequências jurídicas (BRATS, 2014).

É de se notar que todos os fármacos apresentam, em diversas apresentações e formulações, efeitos adversos e colaterais em comum como: Insônia, dor abdominal, cefaléia, taquicardia, dores gastrointestinais, redução do apetite, mania, depressão, tendência à agressividade, excessiva sonolência, desordens psiquiátricas e hipertensão. Ainda em alerta terapêutico dado por ANVISA (2014) o uso destes medicamentos pode causar na faixa etária de 14 a 64 anos os eventos graves envolveram acidente vascular encefálico, instabilidade emocional, depressão, pânico, hemiplegia, espasmos, psicose e tentativa de suicídio.

Existe ainda, no alerta terapêutico a observação para administração destes fármacos, onde o metilfenidato pode diminuir o efeito de medicamentos anti-hipertensivos. Pode também inibir o metabolismo de anticonvulsivantes, anticoagulantes cumarínicos e antidepressivos tricíclicos, aumentando o efeito destes medicamentos. A dose deverá ser ajustada em caso de terapia concomitante.

\section{Conclusão}

Conclui-se que existe uma sazonalidade bimestral na venda destes fármacos com aumento no segundo semestre. Com prevalência de metilfenidato de $10 \mathrm{mg}$ de 30 e 60 comprimidos. Observa-se ainda que a maioria das prescrições seja realizada por especialistas. Existe baseado na literatura uma tendência à medicalização e a utilização destes medicamentos como ampliadores cognitivos.

Sugere-se a ampliação do número de estabelecimentos na cidade. Com a possível descrição da idade e sexo dos usuários para o calculo de DDD e análise da utilização destes medicamentos pela população.

\section{Referências}

ANVISAa. Portaria $n^{\circ}$ 6, de 29 de janeiro de 1999. Aprova a Instrução Normativa da Portaria SVS/MS no 344 de 12 de maio de 1998 que instituiu o Regulamento Técnico das substâncias e medicamentos sujeitos a controle especial. Acesso em: 22 out. 2016

ANVISAb. METILFENIDATO: Indicações terapêuticas e reações adversas. São Paulo: Núcleo de Farmacovigilância do Centro de Vigilância Sanitária da Secretaria de Estado da Saúde de São Paulo, 2013. 2 p. 
ANVISAc. Prescrição e consumo de metilfenidato no Brasil: identificando riscos para o monitoramento e controle sanitário. Boletim de Farmacoepidemiologia do SNGPC. Brasília, p. 1-14. 12 dez. 2012.

BATISTELA, Silmara et al. Methylphenidate as a cognitive enhancer in healthy young people. Dement Neuropsychol, São Paulo, v. 02, n. 10, p.134-142, 10 jun. 2014.

BRANT, Luiz Carlos; CARVALHO, Tales Renato Ferreira. Metilfenidato: medicamento gadget da contemporaneidade. Comunicação Saúde Educação, Belo Horizonte, v. 42, n. 16, p.423-436, 12 jul. 2012.

BRATS. Metilfenidato no tratamento de crianças com transtorno de déficit de atenção e hiperatividade. 23. ed. Brasília: ANVISA, 2014.

CALIMAN, Luciana Vieira; RODRIGUES, Pedro Henrique Pirovani. a experiência do uso de metilfenidato em adultos diagnosticados com tdah. Psicologia em Estudo, Maringá, v. 01, n. 19, p.125-134, 14 mar. 2014.

CARLINI, Elisaldo A. et al. Metilfenidato: influência da notificação de receita A (cor amarela) sobre a prática de prescrição por médicos brasileiros. Rev. Psiq. Clín, São Paulo, v. 01, n. 30, p.120, 06 jan. 2003.

CRF-SP. MANUAL DE ORIENTAÇÃO AO FARMACÊUTICO: ASPECTOS LEGAIS DA DISPENSAÇÃO. São Paulo: Secretaria dos Colaboradores SÃo Paulo, 2017.

FERREIRA, Pedro Eduardo Nader. Autoprescrição de medicamentos e falta de carimbo na receita. Brasilia: CFM, 2014

FREESE, Luana et al. Non-medical use of methylphenidate: a review. Trends Psychiatry Psychother., Porto Alegre, v. 02, n. 34, p.110-115, 03 abr. 2012.

JABOINSKI, Juliana et al. Exposure to methylphenidate during infancy and adolescence in nonhuman animals and sensitization to abuse of psychostimulants later in life: a systematic review. Trends Psychiatry Psychother, Porto Alegre, v. 03, n. 37, p.107-117, set. 2015.

MASÍAS-ARIAS, Lubeth; FIESTAS-TEQUE Luis. Psicosis desencadenada por metilfenidato en un adolescente con trastorno de déficit de atención e hiperactividad. Rev Neuropsiquiatr, Lima, v. 03, n. 77, p.175-178, 16 mar. 2014.

MATTOS, Paulo. Lisdexamfetamine dimesylate in the treatment of attention-deficit/ hyperactivity disorder: pharmacokinetics, efficacy and safety in children and adolescents. Rev Psiq Clín., Rio de Janeiro, v. 9, n. 41, p.34-39, 12 maio 2014.

MAGALHÃES, Fábio Henrique Gomes. Tecnologias Cognitivas: Uma Abordagem Geral. In: $4^{\circ}$ SEMINÁRIO INTERNO DE COGNIÇÃO ARTIFICIAL - SICA 2014 - FEEC - UNICAMP, 4., 2014, Campinas. Faculdade de Engenharia Elétrica e Computação. Campinas, 2014. v. 1, p. 15. 
PERINI, Edson et al. Prescription, dispensation and marketing patterns of methylphenidate. Revista de Saúde Pública, [s.1.], v. 48, n. 6, p.873-880, dez. 2014. Fap UNIFESP (SciELO). http://dx.doi.org/10.1590/s0034-8910.2014048005234.

PINEDA, Arroyo. Lisdexanfetamina. Sescam, Castillha, v. 15, n. 8, p.1-2, 05 maio 2014

RAPELLO, Gabriel Victor Guimarães et al. Pulmonary emphysema induced by methylphenidate: experimental study. São Paulo Med J. São Paulo, v. 03, n. 132, p.131-134, 17 maio 2015.

WALYLO, Andréia et al. Avaliação das prescrições médicas de Ritalina® (metilfenidato) 10mg, disponibilizadas no serviço público de saúde no Município de Ponta Grossa, durante o período de janeiro à junho de 2015. Iessa, Ponta Grossa, v. 1, n. 1, p.1-24, jun. 2016.

WESENSTEN, Nancy J. Effects of Modafinil on Cognitive Performance and Alertness During Sleep Deprivation. Current Pharmaceutical Design USA, v. 12, n. 20, p.2457-2471, maio 2006. 